\title{
Quantitative assessment of parkinsonian bradykinesia based on an inertial measurement unit
}

\author{
Houde Dai ${ }^{12^{*}}$, Haijun $\operatorname{Lin}^{3}$ and Tim C Lueth ${ }^{2}$
}

\section{*Correspondence:}

dhd@fjirsm.ac.cn

${ }^{1}$ Quanzhou Institute

of Equipment Manufacturing,

Fujian Institute of Research

on the Structure of Matter,

Chinese Academy

of Sciences, Quanzhou, China

Full list of author information

is available at the end of the

article

\begin{abstract}
Background: As the most characteristic feature of Parkinson's disease (PD), bradykinesia (slowness of movement) affects all patients with Parkinson's disease and interferes with their daily activities. This study introduces a wearable bradykinesia assessment system whose core component is composed of an inertial measurement unit.
\end{abstract}

Methods: The system diagram and assessment task were defined in accordance with clinical requirements from neurologists. Based on hand grasping actions, calculations of hand grasping ranges and statistical methods of quantitatively assessing parkinsonian bradykinesia were presented. Seven control subjects and eight patients were tested with this system.

Results: Experimental results show that a calculated bradykinesia parameter (modified mean range, instead of mean and standard deviation of the grasp ranges) correlated well with the evaluations of a neurologist (Pearson's correlation coefficient $r=-0.83, p<0.001)$.

Conclusions: The bradykinesia assessment system was tested on both health subjects and PD patients. The results show that this system has greater correlation with the evaluations by neurologists than other parkinsonian bradykinesia assessment systems. The modified mean range was verified as the major bradykinesia parameter (key indicator). This study is helpful to those who want to use consumer-grade inertial sensors for quantitative assessment of motor symptoms during treatment.

\section{Background}

Parkinson's disease (PD) is a movement disorder, which denotes degenerative and progressive disorder of the central nervous system [1]. Parkinson's disease affects patients in many different ways with a variety of symptoms, such as tremor, bradykinesia, rigidity, and postural instability [2].

Parkinsonian tremor is the most well-known and apparent symptom. However, around $30 \%$ of PD patients are not affected by tremor, while Parkinsonian bradykinesia appears in almost all PD patients [2]. Parkinsonian bradykinesia (slowness of motion) involves difficulties in planning, beginning, and executing movement; and difficulties in performing sequential and simultaneous tasks [3].

In the clinical setting, a series of joint tasks, such as finger tapping, whole hand grasping, and supination-pronation movements of hands, are performed by the patients to

(C) 2015 Dai et al. This article is distributed under the terms of the Creative Commons Attribution 4.0 International License (http:// creativecommons.org/licenses/by/4.0/), which permits unrestricted use, distribution, and reproduction in any medium, provided you give appropriate credit to the original author(s) and the source, provide a link to the Creative Commons license, and indicate if changes were made. The Creative Commons Public Domain Dedication waiver (http://creativecommons.org/publicdomain/ zero/1.0/) applies to the data made available in this article, unless otherwise stated. 
assess the bradykinesia severity. A neurologist assesses the severity of parkinsonian bradykinesia according to clinical ratings $[4,5]$.

The current standard for evaluating parkinsonian bradykinesia is the Unified Parkinson's Disease Rating Scale (UPDRS) [4], a qualitative assessment that is completed by the subjective judgment of neurologists. The symptoms are rated on a scale from 0 to 4 (0: normal; 1: slight; 2: mild; 3: moderate; and 4: severe). However, this experience-based assessment may differ among different examiners [2].

To improve the reliability of assessment, some research groups have focused on quantitative assessment of bradykinesia based on computer systems and motion sensors, such as magnetic sensors, electromagnetic sensors, touch sensors, gyroscopes, and accelerometers [5-18]. An overview of recent approaches in parkinsonian bradykinesia assessment is given in Table 1. Based on the examiner's eyesight or sensors, different bradykinesia parameters were adopted. For example, Niazmand et al. used two metal contact pieces, which worked as a touch sensor, to measure the interval of finger taps. However, this self-made touch sensor had long wires and had big live contact pieces [7].

Most recent studies are based on MEMS (micro-electro-mechanical system) inertial sensors [5-18]. Accelerometers, gyroscopes, magnetometers, and their combinations, i.e. inertial measurement units, are the fundamental inertial sensors [19].

Salarian et al. presented a gyroscope-based ambulatory system for quantitative assessment of wrist bradykinesia. There was a significant Pearson's correlation $(r=-0.73$ to $-0.83, p=0.001$ ) between the estimated bradykinesia parameters (range, amplitude, and periods of movements) and UPDRS bradykinesia scores [6]. Kim et al. quantified bradykinesia during finger taps by using a gyroscope. RMS (root-mean-square) velocity, RMS angle, and the estimated power around the dominant frequency were correlated well with clinical finger tapping scores $(r=-0.73$ to $-0.80, p=0.001)$ [8]. Heldman et al. presented a leg assessment system of PD by using inertial sensors, with an average

Table 1 Summary of bradykinesia assessment methods

\begin{tabular}{|c|c|c|c|}
\hline Method or research & Joint; task & Angle measure & Parameters \\
\hline Examiners' assessment & $\begin{array}{l}\text { Hand; grasps, } P-S^{a} / \text { fingers; } \\
\text { taps }\end{array}$ & Visual by the neurologist & Range, frequency \\
\hline Salarian [6] & Wrist; - & Gyroscope & $\begin{array}{l}\text { Range, amplitude, dura- } \\
\text { tions }\end{array}$ \\
\hline Niazmand [7] & Fingers; taps & Metal contact pieces & $\begin{array}{l}\text { Average and standard } \\
\text { deviation of the durations }\end{array}$ \\
\hline Kim [8] & Fingers; taps & Gyroscope & $\begin{array}{l}\text { RMS velocity and angle, } \\
\text { PSD }\end{array}$ \\
\hline Espay [5] & Fingers; taps & Gyroscope & Speed, amplitude, rhythm \\
\hline Heldman [9] & Foot; toe-taps, leg agility & Gyroscope & $\begin{array}{l}\text { RMS of angular velocity and } \\
\text { angle }\end{array}$ \\
\hline $\begin{array}{l}\text { Heldman [10], Kinesia } \\
\text { HomeView }^{\text {TM }}[14]\end{array}$ & $\begin{array}{l}\text { Hand; grasps, } P-S^{a} / \text { fingers; } \\
\text { taps }\end{array}$ & Gyroscope, accelerometer & Range, frequency \\
\hline Printy [11] & Finger; taps/hand; $\mathrm{P}-\mathrm{S}^{\mathrm{a}}$ & Gyroscope, accelerometer & $\begin{array}{l}\text { Frequency, amplitude, } \\
\text { rhythm }\end{array}$ \\
\hline Dunnewold [12] & Wrist; Taps & Accelerometer & Tap rate, durations \\
\hline Zwartjes [13] & $\begin{array}{l}\text { Wrist/thigh/foot/sternum; } \\
\text { taps }\end{array}$ & Accelerometer & $\begin{array}{l}\text { Range and minimum of } \\
\text { acceleration, durations, }\end{array}$ \\
\hline
\end{tabular}

$P-S^{a}$ Pronation-Supination, RMS root mean square and $P S D$ power spectral density. 
correlation coefficient of 0.86 [9]. Heldman et al. also developed a hand bradykinesia assessment system, whose correlation coefficient is 0.67 [10]. Printy et al. presented a smart phone application for quantifying finger bradykinesia $(r=-0.48, p=0.04)$ [11]. Dunnewold et al. tested subjects with wrist taps $(r=-0.61$ to -0.87$)$ [12]. Zwartjes et al. designed an accelerometer-based ambulatory monitoring system to classify bradykinesia severity simultaneously $(r=-0.57$ to -0.71$)$ [13].

Currently, there has been no unique parameter for parkinsonian bradykinesia. The parkinsonian bradykinesia assessment systems provide multiple parameters such as range, frequency, and velocity as bradykinesia parameters [5-18]. Thus, it is difficult for doctors or patients to judge the bradykinesia severity. For the patients and doctors, a single parameter with the highest correlation with the UPDRS bradykinesia scores is preferable. In addition, some of these systems have large dimensions or complex operations. Furthermore, some of their assessment tasks are difficult for old people to perform.

Bradykinesia encompasses slowness, decreased movement amplitude, and dysrhythmia. It means the inability to generate maximum speed, power, or force. These movement tasks are suitable to be tracked by MEMS inertial sensors [20]. Therefore, the goal of this study was to develop a portable system for use both inside and outside the operating room based on the newly developed MEMS inertial sensors [18, 19]. In addition, we also defined a dominant bradykinesia parameter (key indicator) to represent the severity of parkinsonian bradykinesia. A preliminary version of this paper has been reported [18].

\section{Methods}

In this section, the system diagram of the bradykinesia assessment system is firstly introduced, and the preceding methods and prototype implementation are presented.

\section{System diagram and assessment task}

Figure 1 shows the system diagram of the bradykinesia assessment system. The wired communication was adopted due to the requirements of the operating room. The sensor signals from the command module are sent to the computer for further processing [21]. The finger's activity is tracked by an inertial measurement unit (IMU) on the top side of the patient's middle finger, which includes a three-axis gyroscope and a three-axis accelerometer.

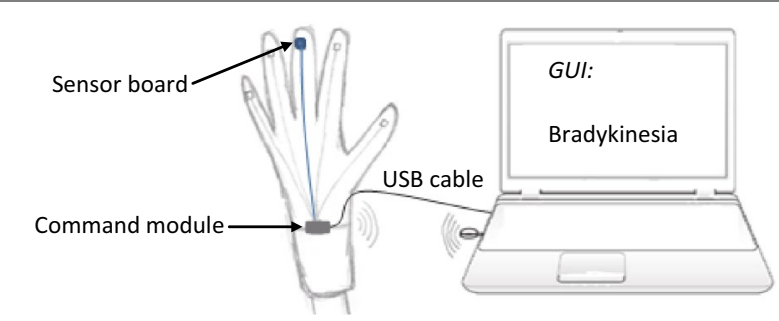

Figure 1 System diagram of the bradykinesia assessment system. The command module, which includes a microcontroller and serial-USB interface, acquires inertial sensor data and sends them to the computer. Doctors can operate the system and view the assessed bradykinesia scores, which are displayed on the graphical user interface (GUI). Additional wireless communication can be adopted for further applications. 
A 10-second whole-hand grasp task was chosen as the assessment action after discussion with neurologists [18]. A single closing and opening action of the fingers is regarded as a grasp cycle. The subject is required to grasp with the greatest possible frequency and range for $10 \mathrm{~s}$.

\section{Processing method}

The hand grasping angles obtained from patients with mild bradykinesia have a consistent amplitude and frequency, and appear sinusoidal. However, hand grasping angles of patients with severe bradykinesia have much lower and more inconsistent amplitudes and frequencies. Speeds, amplitudes, halts, hesitations, and any decline in amplitude are evaluated.

In order to acquire orientation values in real time, a complementary filter, i.e. the direction cosine matrix method (DCM), was adopted [22].

DCM is another way, other than Euler angles, to construct a rotation matrix. The traditional Euler angles have drawbacks such as a gimbal lock. The six-axis DCM algorithm is based on an IMU. The gyroscope is used as the primary source of orientation information. The accelerometer is used for roll-pitch drift correction because it has no drift over time. Only the gravity vector of the accelerometer is used for the drift detection. As shown in Figure 2, a proportional plus integral (PI) controller is used for adjusting the drifts [23]. Each of the rotational drift correction vectors (roll and pitch) is multiplied by weights and fed to a PI feedback controller.

As a control loop feedback mechanism (controller) widely used in industrial control systems, the PI controller calculates an error value as the difference between a measured process variable and a desired set-point. The controller attempts to minimize the error by adjusting the process through the use of a manipulated variable.

After that, the drift adjustments are added to the gyroscope vectors to produce corrected gyroscope vectors. The outputs of the algorithm are three-dimensional angles (orientation).

The grasping ranges $\left(\alpha_{\mathrm{PP}}\right.$ or $\left.\varphi\right)$ are the three-dimensional peak-to-peak values, which are calculated separately, during grasping cycles of a bradykinesia task [10]. The

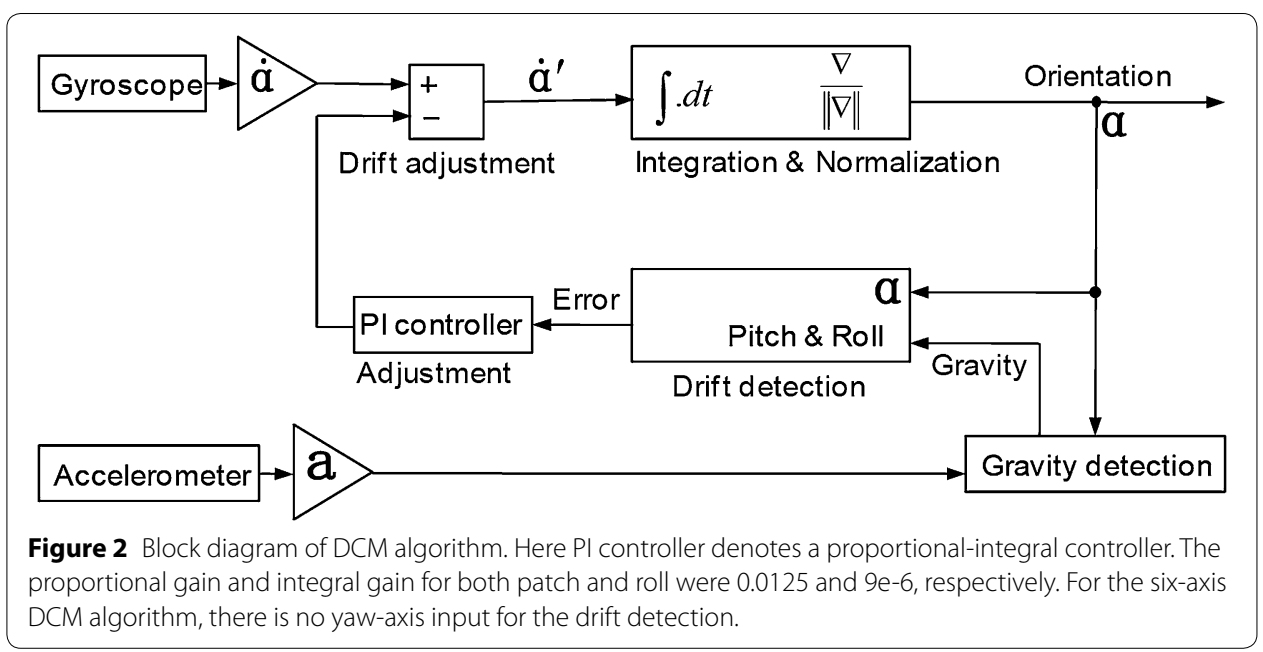


combined triple-axis grasping range $(|\varphi|)$ is the sum of the three-axis grasping ranges $(\varphi)$.

The number of peak-to-peak angle values during the 10-s assessment period is related to the dominant frequency of hand grasps. The mean value of peak-to-peak angle values (grasp ranges) represents the amplitude of bradykinesia. The standard deviation (SD) value of grasp ranges represents the change of amplitude during the grasping task. According to the requirements of neurologists, the mean and SD of the grasp ranges represent the severity of a parkinsonian bradykinesia.

Figure 3 explains the peak-detection method during bradykinesia quantification. There are five hand-grasping cycles in this figure. A peak-detection algorithm could be used to calculate peak-to-peak angle values (ranges 1-5: $|\varphi|_{1}-|\varphi|_{5}$ ).

The flowchart of the peak-detection algorithm is shown in Figure 4. By proceeding through the data starting at zero, the peak detection algorithm tracks the minima and the maxima of the hand-grasping cycles [24].

Tremors occur during the bradykinesia assessment task and it is difficult to separate them in the frequency domain. An angular displacement threshold, for example $\pm 20^{\circ}$ for both maximum and minimum, can be used to remove the unnecessary peak points. For most subjects, the grasp ranges are greater than 20 degrees, so the threshold was fixed at $20^{\circ}$.

The mean and SD values of hand grasping ranges are easy to calculate by using statistical methods. Equations 1 and 2 show the mathematical formulas used to determine $(\overline{|\varphi|})$ and $\sigma_{\varphi}$ :

$$
\begin{aligned}
\overline{|\boldsymbol{}|} & =\left(\sum_{i=1}^{N}|\boldsymbol{\varphi}|_{i}\right) / N, \\
\sigma_{|\phi|} & =\sqrt{\frac{1}{N-1} \cdot \sum_{i=1}^{N}\left(|\boldsymbol{\varphi}|_{i}-\overline{|\boldsymbol{}|}\right)^{2}},
\end{aligned}
$$

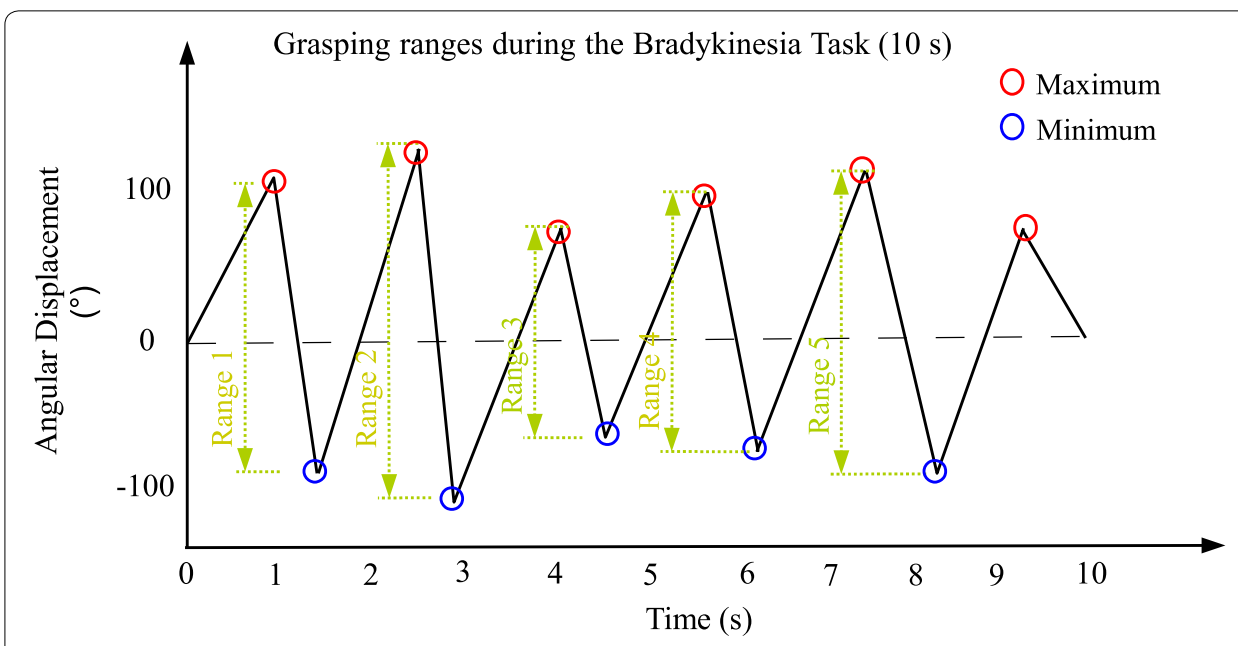

Figure 3 Peak detection for grasping ranges in a bradykinesia task. After the maximum and minimum points of the grasping ranges are found, the grasping ranges during the one-time bradykinesia task are acquired. 


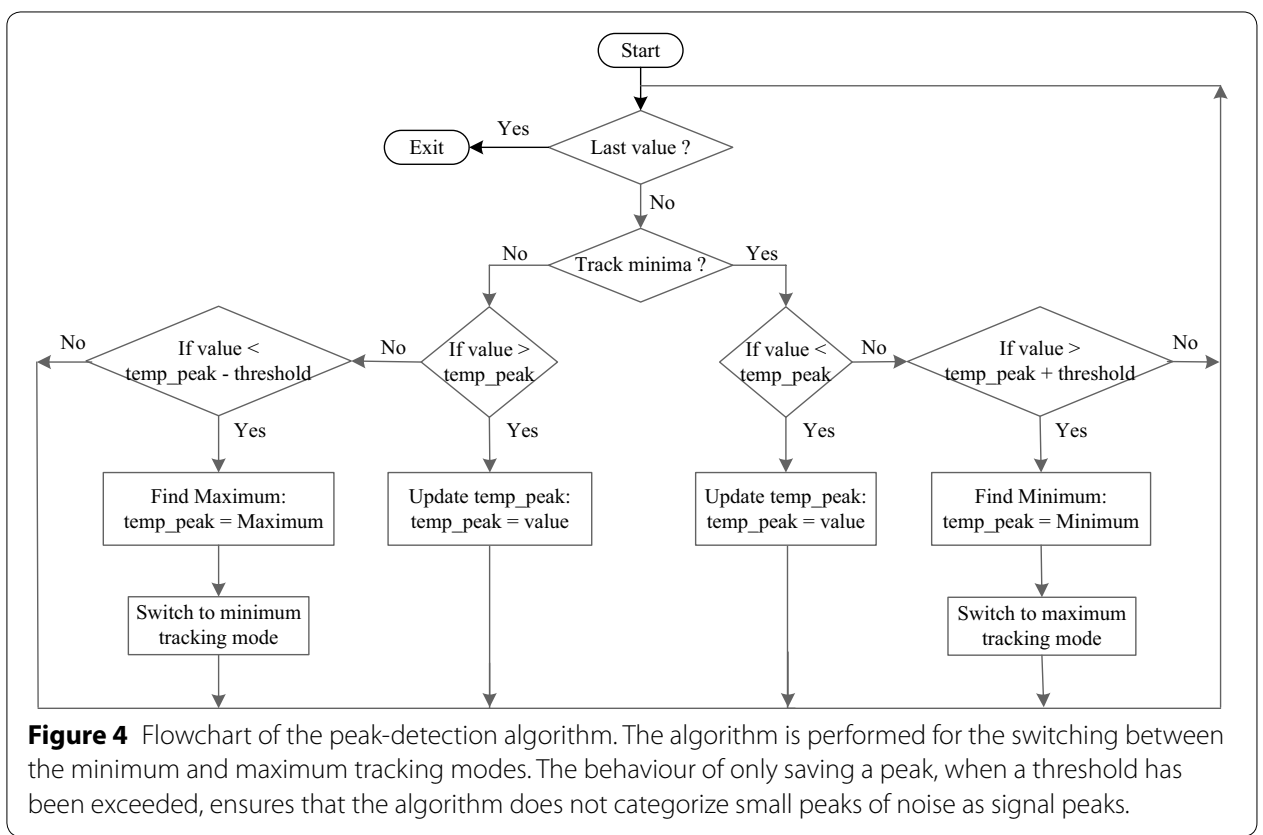

where $|\varphi|_{i}$ is the combined hand grasping range (peak-to-peak values) in a single grasp cycle; and $N$ is the number of hand grasping cycles in a 10-s bradykinesia task.

The dominant frequency of hand grasps is calculated by using the Fast Fourier Transform (FFT) method from the grasping ranges.

In addition, the difficulty in selecting or activating motor programs in the central nervous system may result in akinesia (inability to initiate movement) in the patient's daily life. Akinesia (absence of movement) during the bradykinesia task is the delay (action time) of the patient in starting the assessment task after receiving the instruction from the examiner [25]. In this study, the effect of akinesia on the parkinsonian bradykinesia was not considered.

\section{Prototypical realization}

The system implementation of the bradykinesia assessment system is shown in Figure 5.

The full ranges of the IMU in the parkinsonian bradykinesia assessments are listed as follows [14]:

- Angular velocity: $\pm 2,000^{\circ} / \mathrm{s}$ (degrees per second or dps) in three dimensions;

- Acceleration: $\pm 4 g$ in each axis, here $g$ is the gravitational acceleration $\left(1 g=9.8 \mathrm{~m} / \mathrm{s}^{2}\right)$.

MPU6050 (Invensense Inc., USA), an inertial measurement unit which is used for motion tracking, was chosen in this project. The axes of the accelerometer and gyroscope are the same [26]. Thus adjustment of the axes before sensor fusion is not necessary. The final implementation of the bradykinesia assessment system is shown in Figure 6. A shielded four-pin cable was used to connect the sensor board to the command module.

The USB port of the computer provided the power supply $(+5 \mathrm{~V})$ for the command module. The IMU in the sensor board was connected to the microprocessor via the IIC 

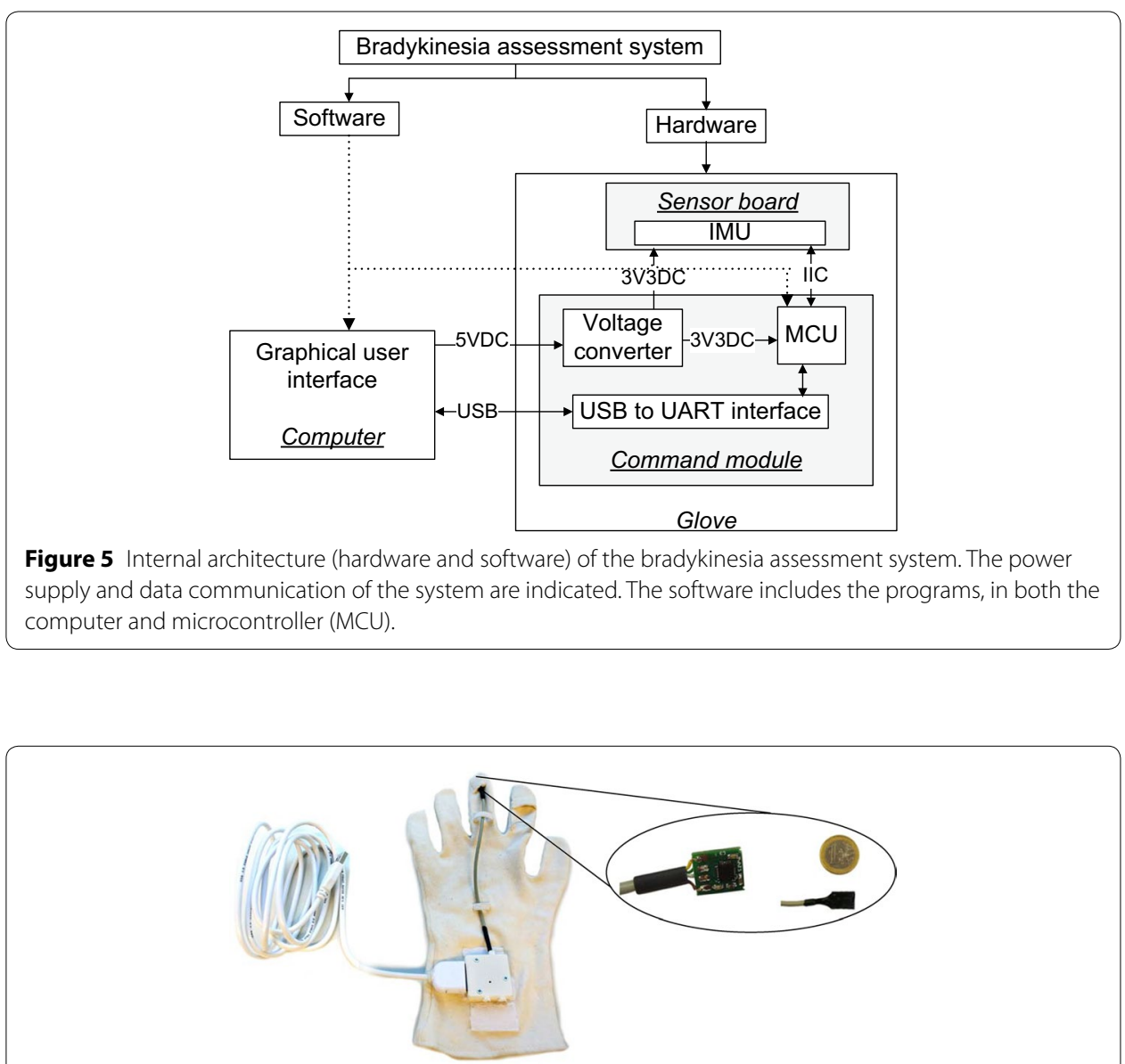

Figure 6 Photos of the prototype for the bradykinesia assessment system. The command module was $34 \mathrm{~mm} \times 32 \mathrm{~mm} \times 7 \mathrm{~mm}$. Both the command module and sensor board had indirect contact with the human body. The sensor board (dimensions: $14 \mathrm{~mm} \times 11 \mathrm{~mm} \times 2 \mathrm{~mm}$ ) is shielded by a synthetic rubber coating material.

(pronounced I-two-C) serial interface. The microprocessor sampled the IMU data at $100 \mathrm{~Hz}$.

The data transmission, signal processing, and GUI of the bradykinesia assessment system were carried out on a computer using LabVIEW 2011 (National Instruments Corp., USA).

The operation of the bradykinesia assessment system is shown in Figure 7.

\section{Experiment of bradykinesia assessment}

In this section, the clinical experiment and the results are presented. The prototype was tested with both healthy controls and PD patients.

\section{Hypothesis}

We expected the correlations of bradykinesia parameters (mean and SD values of amplitude, and grasp frequency) between the bradykinesia assessment system and clinical ratings to meet the following requirement [5-18]:

$H_{0}$ : Pearson's correlation coefficient $r=0.79 ; H_{1}: r \neq 0, \alpha=0.01$ (2-tailed). 


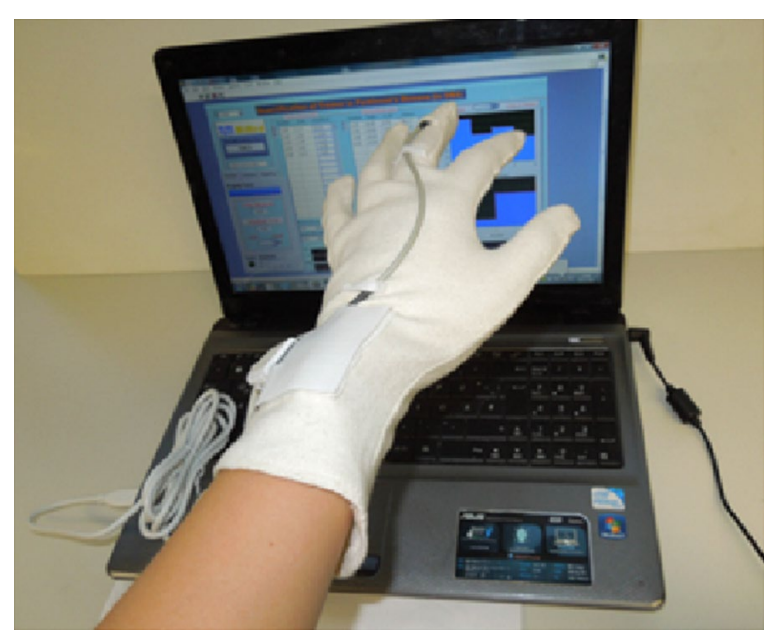

Figure 7 Bradykinesia assessment system. This system is based on a computer, a textile glove, and a command module.

\section{Materials}

- A bradykinesia assessment system;

- A computer installed with the GUI of the bradykinesia assessment system (based on LabVIEW, National Instruments Corp., USA) and MATLAB (Mathworks Inc., USA).

\section{Experiment setup}

Seven healthy controls (average age: $57.1 \pm 21.1$ years) and nine PD patients (average age: $72.8 \pm 10.0$ years) were required to execute hand grasping actions as widely and quickly as possible for $10 \mathrm{~s}$, and repeat the assessment task two or three times for each subject. The first time of each assessment task was discarded for training. However, a severe PD patient (UPDRS bradykinesia score $D=4$ ) was unable to perform the bradykinesia task. The patients were tested $24 \mathrm{~h}$ after off medication and without deepbrain stimulation. The one-time whole-hand grasping task included several grasp cycles, which was the number of peak-to-peak ranges.

An IMU, attached to the middle finger, was used to measure the angular displacement of the middle finger during the bradykinesia assessment task. The bradykinesia parameters, calculated by the assessment system and used as the severity features of bradykinesia, were compared to the ratings of a neurologist [18].

The mean and SD values of the grasp ranges represent the severity of the parkinsonian bradykinesia. However, we inferred that the dominant frequency also has a strong correlation with the UPDRS score; thus a modified mean range, which equals the product of the dominant frequency and mean range during a single bradykinesia assessment task, was chosen as the dominant bradykinesia parameter.

The UPDRS scores for healthy controls are judged as zero, although their assessed values may deviate from normal values.

Our research group previously adopted finger tapping as assessment task. However, neurologists suggested that we adopt whole-hand grasping. During clinical experiments, therefore, the patient and control subjects were required to perform both finger tapping 
and whole-hand grasping actions. Most patients and control subjects preferred wholehand grasping as it was easier for them to perform.

\section{Discussion}

Figure 8 shows three 10-s waveforms of hand grasps and their power spectral density (PSD) figures. Figure 8a shows the waveforms from a healthy subject, while Figure $8 b, c$ show the waveforms from PD patients.

In addition, other motor symptoms also appeared in the hand grasp tasks. As shown in Figure $8 \mathrm{~b}$, action tremor appeared among some patients during the bradykinesia assessment task. As shown in Figure 8c, akinesia (difficulty initiating movement) appeared in a patient with severe bradykinesia [27]. It can be seen from Figure $8 \mathrm{c}$ that there was a delay time before he performed hand grasps continuously. As Figure $8 \mathrm{c}$ shows, the akinesia affects the cycles of hand grasps, thus the dominant frequency was reduced.

Table 2 shows the calculated bradykinesia parameters of this experiment and the Pearson's correlation coefficients $(r)$ between these parameters and the judgment of the neurologist (UPDRS scores $D$ ). Figure 9 shows the relations between the judgments of a neurologist and the UPDRS bradykinesia parameters.

The seven control subjects had a dominant frequency at $1.19 \pm 0.08 \mathrm{~Hz}$ and mean range at $24,246.7^{\circ} \pm 34.2^{\circ}$. For patients with slight bradykinesia, the dominant frequency was at $1.17 \pm 0.17 \mathrm{~Hz}$ and mean range at $195.0^{\circ} \pm 37.6^{\circ}$, while the patients with mild and severe bradykinesia were $0.54^{\circ} \pm 0.16 \mathrm{~Hz}$ and $188.4^{\circ} \pm 60.2^{\circ}$ respectively.

As Table 2 shows, the patients with severe symptoms (Patients 6-8) executed grasping cycles with a lower modified mean range $\left(187.1^{\circ} \pm 53.9^{\circ}\right)$ and dominant frequency $(0.60 \pm 0.30 \mathrm{~Hz})$, while the modified mean range and dominant frequency from control subjects were $246.3^{\circ} \pm 36.2^{\circ}$ and $1.2^{\circ} \pm 0.1^{\circ}$ respectively. The dominant frequency of hand grasps and the modified mean range had the highest correlations with the UPDRS bradykinesia score $(r=-0.79$ and -0.83 respectively; $p<0.001)$. The correlations were greater than 0.81 , which was defined by the Kinesia system, thus $H_{1}$ was accepted [10].
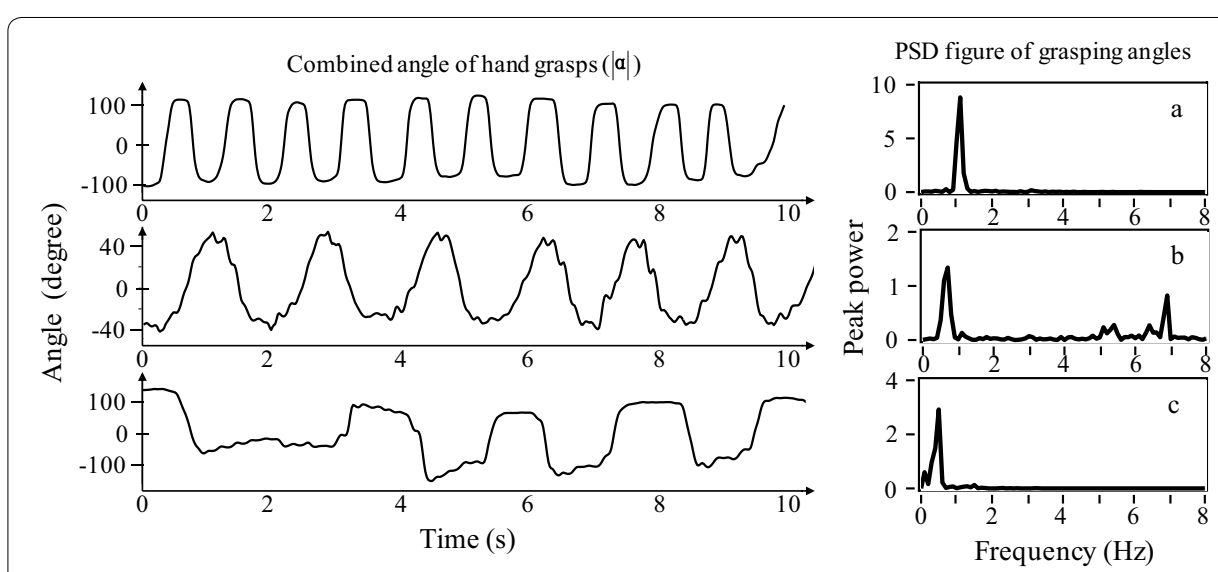

Figure 8 Ten-second hand grasps and their PSD figures. a 72 year old control subject; b 82 year old PD patient with tremor and bradykinesia, UPDRS bradykinesia score $D=1 ; \mathbf{c} 86$ year old PD patient, UPDRS bradykinesia score $D=3$. The peak powers in the figure were calculated based on the power estimation around the dominant frequency and with a weighted scale. 
Table 2 Clinical experiment results

\begin{tabular}{|c|c|c|c|c|c|}
\hline $\begin{array}{l}\text { Subjects; } \\
\text { age (years) }\end{array}$ & $\begin{array}{l}\text { Dominant } \\
\text { frequency }\end{array}$ & $\begin{array}{l}\text { Mean } \\
\text { range }\end{array}$ & SD ranges & $\begin{array}{l}\text { Modified } \\
\text { mean range }\end{array}$ & $\begin{array}{l}\text { UPDRS } \\
\text { score }(D)\end{array}$ \\
\hline Control $1 ; 72$ & $1.11 \mathrm{~Hz}$ & $202.9^{\circ}$ & $7.6^{\circ}$ & $220.5 \% / \mathrm{s}$ & 0 \\
\hline Control 2; 46 & $1.29 \mathrm{~Hz}$ & $255.4^{\circ}$ & $11.5^{\circ}$ & $329.5 \% / \mathrm{s}$ & 0 \\
\hline Control 3; 29 & $1.10 \mathrm{~Hz}$ & $289.8^{\circ}$ & $19.8^{\circ}$ & $318.8^{\circ} / \mathrm{s}$ & 0 \\
\hline Control 4; 34 & $1.30 \mathrm{~Hz}$ & $237.2^{\circ}$ & $10.7^{\circ}$ & $310.7 \% / \mathrm{s}$ & 0 \\
\hline Control 5; 61 & $1.18 \mathrm{~Hz}$ & $270.9^{\circ}$ & $11.5^{\circ}$ & $319.7^{\circ} / \mathrm{s}$ & 0 \\
\hline Control 6; 76 & $1.16 \mathrm{~Hz}$ & $246.6^{\circ}$ & $8.6^{\circ}$ & $286.0^{\circ} / \mathrm{s}$ & 0 \\
\hline Control $7 ; 82$ & $1.22 \mathrm{~Hz}$ & $195.8^{\circ}$ & $8.2^{\circ}$ & $238.5 \% / \mathrm{s}$ & 0 \\
\hline Patient 1; 82 & $0.88 \mathrm{~Hz}$ & $241.6^{\circ}$ & $10.2^{\circ}$ & $212.6 \% \mathrm{~s}$ & 1 \\
\hline Patient 2; 73 & $1.34 \mathrm{~Hz}$ & $162.6^{\circ}$ & $20.0^{\circ}$ & $226.0 \% / \mathrm{s}$ & 1 \\
\hline Patient 3; 76 & $1.40 \mathrm{~Hz}$ & $182.0^{\circ}$ & $11.7^{\circ}$ & $263.9 \% / \mathrm{s}$ & 1 \\
\hline Patient 4; 75 & $1.13 \mathrm{~Hz}$ & $160.8^{\circ}$ & $16.8^{\circ}$ & $184.9^{\circ} / \mathrm{s}$ & 1 \\
\hline Patient 5; 56 & $1.16 \mathrm{~Hz}$ & $247.5^{\circ}$ & $17.7^{\circ}$ & $309.4 \% \mathrm{~s}$ & 1 \\
\hline Patient 5; 56 & $1.11 \mathrm{~Hz}$ & $236.4^{\circ}$ & $22.1^{\circ}$ & $267.1 \% / \mathrm{s}$ & 1 \\
\hline Patient 6;61 & $1.15 \mathrm{~Hz}$ & $180.7^{\circ}$ & $27.0^{\circ}$ & $216.8^{\circ} / \mathrm{s}$ & 1 \\
\hline Patient 6; 61 & $0.65 \mathrm{~Hz}$ & $87.1^{\circ}$ & $5.5^{\circ}$ & $61.0 \% \mathrm{~s}$ & 2 \\
\hline Patient 7; 73 & $0.60 \mathrm{~Hz}$ & $181.7^{\circ}$ & $9.5^{\circ}$ & $109.0 \% / \mathrm{s}$ & 2 \\
\hline Patient $7 ; 73$ & $0.44 \mathrm{~Hz}$ & $213.3^{\circ}$ & $20.2^{\circ}$ & $93.6 \% \mathrm{~s}$ & 2 \\
\hline Patient 8; 86 & $0.42 \mathrm{~Hz}$ & 238.0 & $5.4^{\circ}$ & $100.0 \% / \mathrm{s}$ & 2 \\
\hline Patient 8; 86 & $0.32 \mathrm{~Hz}$ & $221.8^{\circ}$ & $3.6^{\circ}$ & $71.0 \% \mathrm{~s}$ & 3 \\
\hline$r$ & -0.79 & -0.39 & -0.13 & -0.83 & \\
\hline$p$ (2-tailed) & $<0.001$ & 0.10 & 0.59 & $<0.001$ & \\
\hline
\end{tabular}
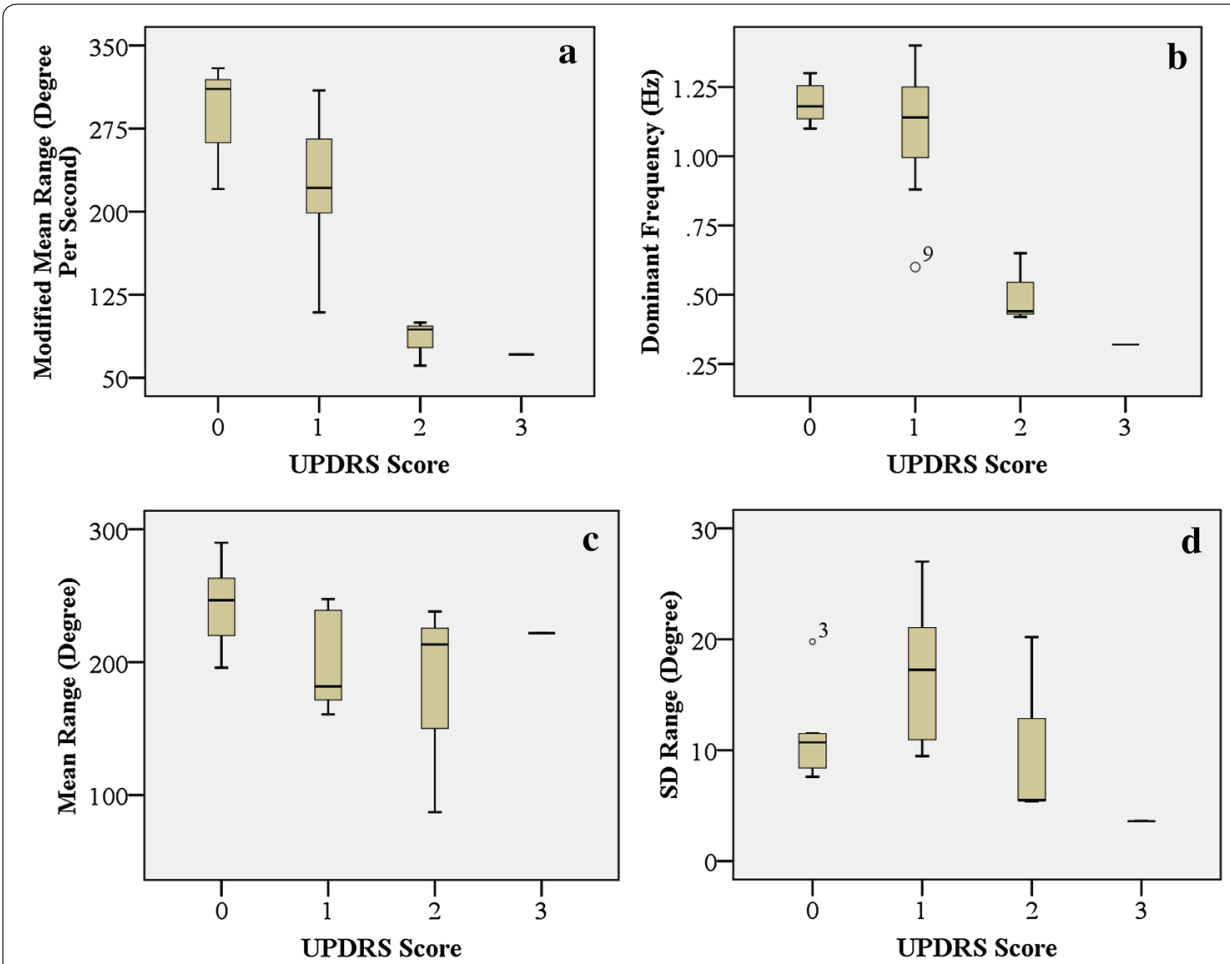

Figure 9 Box-plots displaying the relations between the judgments of a neurologist and the UPDRS bradykinesia parameters. a Modified mean range; b Dominant frequency; c Mean range of the grasping ranges; $\mathbf{d}$ SD of the grasping ranges. The unit of ranges in all plots is degrees. 
However, the mean range of hand grasps had a low correlation with the UPDRS bradykinesia score $(r=-0.39)$. For the SD ranges, there was an even smaller correlation coefficient $(r=-0.13)$ with the UPDRS ratings in this experiment. The mean and SD of the grasping ranges should therefore not be regarded as major bradykinesia parameters.

As Table 2 also shows, the age of a subject may influence her or his performance of hand grasping, as shown by Control subject 1 and Patient 5 , whose mean range deviated from the normal situation. Control subject 1 (72 years) could not perform great amplitudes (mean range $\left.=202.9^{\circ}\right)$ due to her age, while Patient $5(56$ years, UPDRS score $=1)$ performed with a higher dominant frequency $(1.14 \mathrm{~Hz})$ and mean range $\left(242.0^{\circ}\right)$.

As a conclusion, the dominant frequency and modified mean range between the bradykinesia assessment system and a surgeon were significant $(|r| \geq 0.83)$. Thus the modified mean range can be defined as the major parkinsonian bradykinesia parameter.

\section{Conclusions}

A MEMS IMU, which is a typical motion sensor, was employed in a wearable bradykineisa assessment system and performed motion tracking.

Instead of finger tapping for bradykinesia assessment as in most research groups, this study uses whole-hand grasping. Compared with the more common used finger tapping, hand grasping is easy for patients to perform.

There were great differences between the control subjects and patients with parkinsonian bradykinesia, because the average modified mean range of control subjects, patients with slight, and mild or worse bradykinesia were $294.9,240.1$, and $86.92^{\circ} / \mathrm{s}$ respectively.

At the beginning of this study, neurologists considered the mean and SD values of grasping ranges to have an even higher correlation with the bradykinesia scores. According to the results of the clinical experiment, however, the dominant frequency and modified mean range of hand grasps correlated well with the UPDRS ratings (the absolute values of correlation coefficients were 0.79 and 0.83 respectively), while the mean and SD of grasping ranges were 0.39 and 0.13 respectively. As a result, the modified mean range had the highest correlation coefficient with the judgment of the neurologist and can thus be adopted as the major bradykinesia parameter.

Future research will include carrying out the experiment with more PD patients. However, attention must be paid to the data processing methods as concerns the following:

1. Verification and comparison of the different calculation methods of the hand grasping range because calibration procedures, signal filters, and multi-sensor algorithms play a key role in the range calculation [27].

2. As there are no previous studies about the correlation between age and the quantitative parameters of parkinsonian bradykinesia, the repeatability of bradykinesia parameters and effects of the subject's age and physical status (both mental and physical factors) on the bradykinesia parameters should be investigated [28].

3. Effects of PD's other symptoms, such as tremor, akinesia, and motor fluctuations [29, 30], on the bradykinesia parameters.

4. Correlations between the features were taken into consideration together with the clinical evaluations of bradykinesia provided by several neurologists, in order to assess the robustness of the method proposed. 
5. The angular displacement threshold was fixed at 20 degrees. In future works, we can set an automatic threshold which is proportional to the grasp ranges.

\section{Authors' contributions}

HD conceived, designed and implemented the system, acquired the data and drafted the manuscript. Prof. TCL contributed to valuable discussions and suggestions. HL provided suggestions for the signal processing algorithms. All authors read and approved the final manuscript.

\section{Author details}

${ }^{1}$ Quanzhou Institute of Equipment Manufacturing, Fujian Institute of Research on the Structure of Matter, Chinese Academy of Sciences, Quanzhou, China. ${ }^{2}$ Institute of Micro Technology and Medical Device Technology, Faculty of Mechanical Engineering, Technische Universitaet Muenchen, Boltzmannstr. 15, 85748 Garching, Germany. ${ }^{3}$ College of Polytechnic, Hunan Normal University, Changsha, China.

\section{Acknowledgements}

The authors would like to thank Prof. Dr. Tonn and Dr. J. H. Mehrkens from the Department of Neurosurgery, University Hospital Grosshadern, (LMU Muenchen) for their work in this project. The authors would also like to thank all the test subjects for their kind support.

\section{Compliance with ethical guidelines}

\section{Competing interests}

The authors declare that they have no competing interests.

Received: 13 January 2015 Accepted: 16 June 2015

Published online: 12 July 2015

\section{References}

1. Jankovic J. Parkinson's disease: clinical features and diagnosis. J Neurol Neurosurg Psychiatry. 2008;79(4):368-76.

2. Chaudhuri KR, Ondo WF. Handbook of movement disorders. London: Springer Healthcare Ltd; 2011.

3. Rothwell JC, Thompson PD, Hallett M. Pathophysiology of bradykinesia in Parkinson's disease. Brain. 2001;123:2121-46

4. Ramaker C, Marinus J, Stiggelbout AM, Van Hilten BJ. Systematic evaluation of rating scales for impairment and disability in Parkinson's disease. Mov Disord. 2002;17(5):867-76.

5. Espay AJ, Giuffrida JP, Chen R, Payne M, Mazzella F, Dunn E, et al. Differential response of speed, amplitude, and rhythm to dopaminergic medications in Parkinson's disease. Mov Disord. 2011;26(14):2504-8.

6. Salarian A, Russmann H, Wider C, Burkhard PR, Vingerhoets FJ, Aminian K. Quantification of tremor and bradykinesia in Parkonson's disease using a novel ambulatory monitoring system. IEEE Trans Biomed Eng. 2007;54:313-22.

7. Niazmand K, Tonn K, Kalaras A, Fietzek UM, Mehrkens JH, Lueth TC. Quantitative evaluation of Parkinson's disease using sensor based smart glove. IEEE Symposium on Computer-Based Medical Systems-CBMS, UK. 2011. p. 1-8

8. Kim JW, Lee JH, Kwon Y, Kim CS, Eom GM, Koh SB, et al. Quantification of bradykinesia during clinical finger taps using a gyroscope in patients with Parkinson's disease. Med Biol Eng Comput. 2011;49:365-71.

9. Heldman DA, Filipkowski DE, Riley DE, Whitney CM, Walter BL, Gunzler SA, et al. Automated motion sensor quantification of gait and lower extremity bradykinesia. IEEE EMBC Conf. 2012. p. 1956-9.

10. Heldman DA, Giuffrida JP, Chen R, Payne M, Mazzella F, Dunn E, et al. The modified bradykinesia rating scale for Parkinson's disease: reliability and comparison with kinematic measures. Mov Disord. 2011;26(10):1859-63.

11. Printy BP, Renken LM, Herrmann JP, Lee I, Johnson B, Knight E, et al. Smartphone application for classification of motor impairment severity in Parkinson's disease. IEEE EMBC. 2014. p. 2686-9.

12. Dunnewold RJ, Jacobi CE, van Hilten JJ. Quantitative assessment of bradykinesia in patients with Parkinson's disease. J Neurosci Methods. 1997;74(1):107-12

13. Zwartjes D, Heida T, van Vugt J, Geelen J, Veltink P. Ambulatory monitoring of activities and motor symptoms in Parkinson's disease. IEEE Trans Biomed Eng. 2010;57(11):2778-86.

14. User's Guides for Kinesia HomeView ${ }^{\text {TM }}$ Kinesia, Ref. No. G392-5001. Great Lakes NeuroTechnologies Inc. USA. 2014. http://www.glneurotech.com/kinesia/products/homeview/. Accessed 11 Jan 2015.

15. Synnott J, Liming C, Nugent CD, Moore G. WiiPD_Objective home assessment of Parkinson's disease using the Nintendo Wii remote. IEEE Trans Inf Technol Biomed. 2012;16(6):1304-12.

16. Takuyuki E, Masaru Y, Harutoshi F, Saburo S. Chapter 9: Novel methods to evaluate symptoms in parkinson's diseaserigidity and finger tapping. In: Dushanova J, editor. Diagnostics and rehabilitation of Parkinson's disease. Croatia: InTech; 2011. p. 191-206.

17. Shima K, Tsuji T, Kan E, Kandori A, Yokoe M, Sakoda S. Measurement and evaluation of finger tapping movements using magnetic sensors. IEEE EMBS Conf. 2008. p. 5628-31.

18. Dai H, DAngelo LT. A portable system for quantitative assessment of parkinsonian bradykinesia during deep-brain stimulation surgery. IEEE Second International Conference on Advances in Biomedical Engineering, Lebanon. 2013. p. 77-80.

19. Shaeffer DK. MEMS inertial sensors: a tutorial overview. IEEE Commun Mag. 2013;51(4):100-9.

20. Khoshnoud F, de Silva CW. Recent advances in MEMS sensor technology—biomedical applications. IEEE Instrum Meas Mag. 2012;15(1):8-14. 
21. Lueth TC, D'Angelo LT, Czabke A. Chapter 4:TUM-AgeTech, a new framework for pervasive medical devices. In: Coronato A, Pietro GD, editors. Pervasive and smart technologies for healthcare: ubiquitous methodologies and tools. New York: IGI Global; 2010. p. 295-321.

22. Edwan E, Zhang JY, Zhou JC, Loffeld O. Reduced DCM based attitude estimation using low-cost IMU and magnetometer triad. 8th Workshop on Positioning Navigation and Communication (WPNC). 2011. p. 1-6.

23. Edwan E, Zhang J, Zhou J, Loffeld O. Reduced DCM based attitude estimation using low-cost IMU and magnetometer triad. 8th Workshop on Positioning Navigation and Communication (WPNC). 2011, p. 1-6.

24. Jordanov VT, Hall DL. Digital peak detector with noise threshold. IEEE Nuclear Science Symposium Conference Record. Durham, USA. 2002;1. p. 140-2.

25. Giovannoni G, Schalkwyk J, Fritz VU, Lees AJ. Bradykinesia akinesia inco-ordination test (BRAIN TEST): an objective computerized assessment of upper limb motor function. J Neurol Neurosurg Psychiatr. 1999;67(5):624-9.

26. Geiger W, Bartholomeyczik J, Breng U, Gutmann W, Hafen M, Handrich E, et al. MEMS IMU for AHRS applications. IEEE/ION Symposium on Position, Location and Navigation. 2008. p. 225-31

27. Madgwick SOH, Harrison AJL, Vaidyanathan A. Estimation of IMU and MARG orientation using a gradient descent algorithm. IEEE ICORR Conf, Zurich. 2011. p. 1-7.

28. Heldman DA, Espay AJ, LeWitt PA, Giuffrida JP. Clinician versus machine: reliability and responsiveness of motor endpoints in Parkinson's disease. Parkinsonism Relat Disord. 2014;20(6):590-5.

29. Patel S, Lorincz K, Hughes R, Huggins N, Growdon J, Standaert D, et al. Monitoring motor fluctuations in patients with Parkinson's disease using wearable sensors. IEEE Trans Inf Technol Biomed. 2009;13(6):864-73.

30. Dai H, Otten B, Mehrkens JH, D'Angelo LT, Lueth TC. A novel glove monitoring system used to quantify neurological symptoms during deep-brain stimulation surgery. IEEE Sens J. 2013;13(9):3193-320.

\section{Submit your next manuscript to BioMed Central and take full advantage of:}

- Convenient online submission

- Thorough peer review

- No space constraints or color figure charges

- Immediate publication on acceptance

- Inclusion in PubMed, CAS, Scopus and Google Scholar

- Research which is freely available for redistribution

Submit your manuscript at 